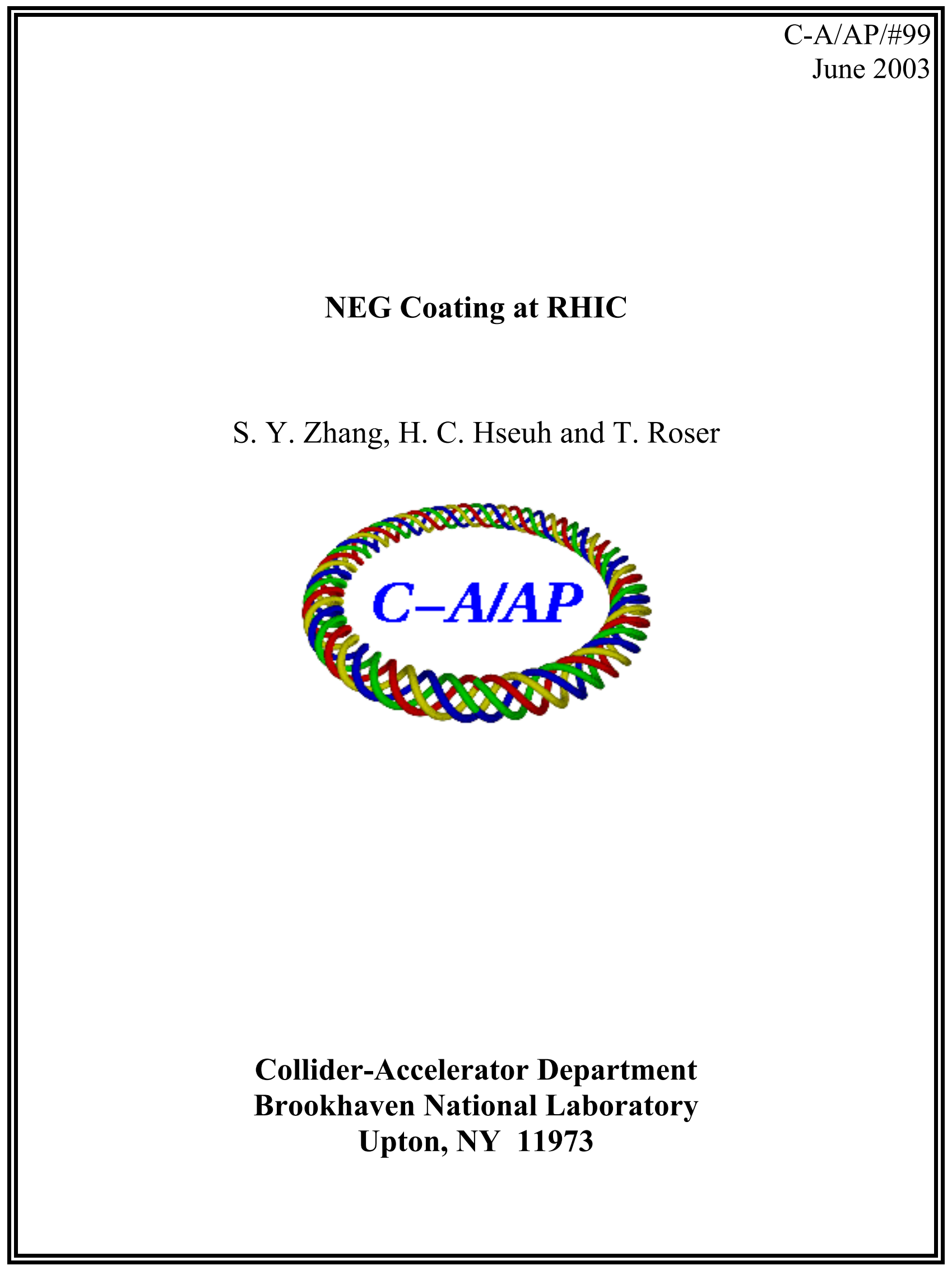




\title{
NEG Coating at RHIC
}

\author{
S.Y. Zhang, H.C. Hseuh, and T. Roser
}

\section{Introduction}

In recent years, NEG (non-evaporable-getter) coating has been developed to improve accelerator vacuum conditions [1]. Based on the principle of getter-strip, which was applied to CERN LEP, and also to BNL Tandem Van de Graaff, the thin film coating on the chamber wall is a non-trivial step forward on several fundamental aspects.

1. NEG coating does not affect machine aperture, unlike the getter-strip.

2. NEG coating turns the chamber wall from outgassing source to pumping surface.

3. Secondary electron yield (SEY) is significantly reduced from the stainless steel wall.

4. Electron stimulated gas desorption rate is reduced.

5. Ion gas desorption rate is also reduced.

Study is still underway to better understand the NEG coating. Some associated problems, such as aging, activation, dust, etc. may need attentions. On the other hand, it has already been successfully applied to several machines, including ESRF, and it is a baseline design for CERN LHC warm section chamber of $8 \mathrm{~km}$ length in total.

The pressure rise at RHIC is caused by not only the electron cloud, but also the beam loss, and perhaps more seriously, the beam halo scraping [2]. Counter measure for electron multipacting is not sufficient, reduction of electron and ion gas desorption rates is also needed. In addition, the experiment background in terms of beam-gas might be improved from the high linearly distributed pumping, which NEG coating can provide. Therefore, it is of interest to explore the application of NEG coating at RHIC.

In this article, the pressure rise concerns at RHIC will be presented. Properties of NEG coating in SEY, electron and ion desorption will be reviewed based on measurements. Complications in applying NEG coating at RHIC will also be discussed.

\section{Pressure rise concerns at RHIC}

RHIC pressure rise at the beam injection may be caused by electron cloud, and the one at transition seems the result of beam halo scraping.

\section{Electron multipacting induced pressure rise}

Electron multipacting caused pressure rise, typically at beam injection, has been observed for proton, deuteron and gold beams in RHIC operation. This type of pressure rise is very sensitive to bunch intensity and bunch spacing, therefore, the situation of 110-bunch injection is much worse than the 55-bunch mode. The intensity threshold at long straight section, i.e., single beam pipe between Q3 and Q4, is lower than the one at interaction straight section with shorter chamber. Pressure rise tends to saturate, with the saturation level approximately proportional to the beam 
intensity. Run-away type of pressure rise had been observed in gold beam run, but it always accompanied by beam loss. It is suspected that the gold beam loss generated positive ions may have neutralized the electron cloud, and hence the saturation limit is raised.

In Run 2003, electron multipacting signals have been detected. Pressure rise was found to coincide with the start of electron multipacting, and the pressure rise is approximately proportional to the amplitude of electron signal.

Solenoid can reduce the electron signal, and in general, reduce the pressure rise [2]. On the other hand, the solenoid effect on the pressure rise has shown some complication, and further study is needed.

\section{Halo scraping caused pressure rise}

In 2003 deuteron-gold run, pressure rise at the transition had raised concern. This pressure rise seems not being caused by electron multipacting, but by beam halo scraping [2].

In fact, the transition pressure rise existed in the 2001 gold-gold run. However, the total beam intensity then was lower than the run 2003, the amplitude of this pressure rise was not large enough to raise concern.

Comparison of the solenoid effect at the experiment STAR, with respect to the electron multipacting induced pressure rise and the transition pressure rise, supports the argument that the transition pressure rise is not caused mainly by electrons.

Fig.1 shows that in proton run, the STAR solenoid effectively suppressed electron multipacting type pressure rise at beam injection.
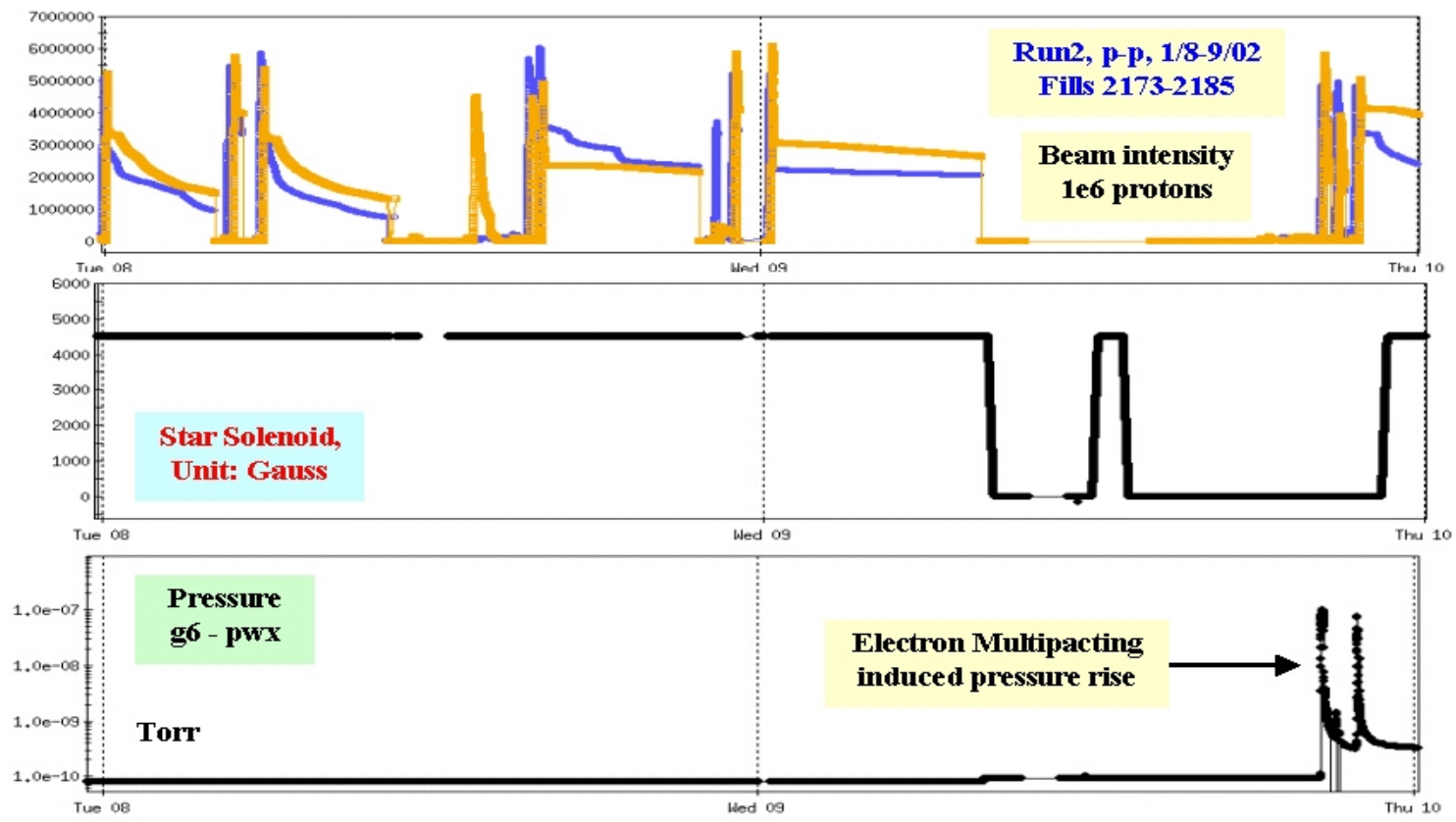

Fig.1: Electron multipacting caused pressure rise was observed at the interaction region IR6, the STAR detector location. Solenoid field suppressed the pressure rise.

Fig.2 shows that in 2001 gold-gold run, the STAR solenoid field did not affect the pressure rise at the transition. 

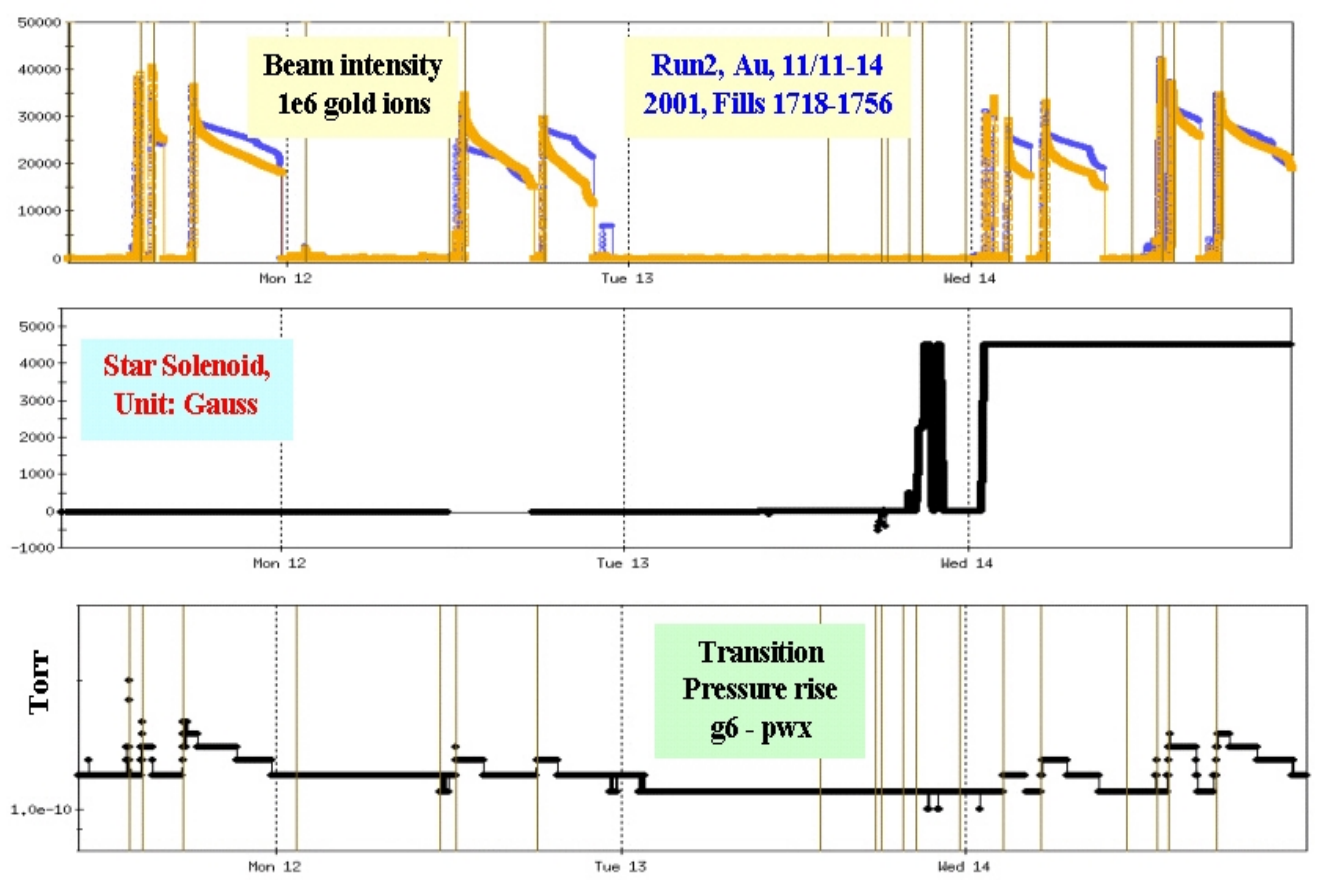

Fig.2: The transition pressure rise was not affected by the solenoid field in 2001 gold-gold run. Brown vertical lines stand for the time of transition.

Another example that beam halo scraping may cause pressure rise is shown in Fig 3 of proton and Fig.4 of deuteron ramps.

Fig. 3 shows that the pressure rise at both the single beam straight section and interaction section started to decrease as the beam acceleration is started [3]..
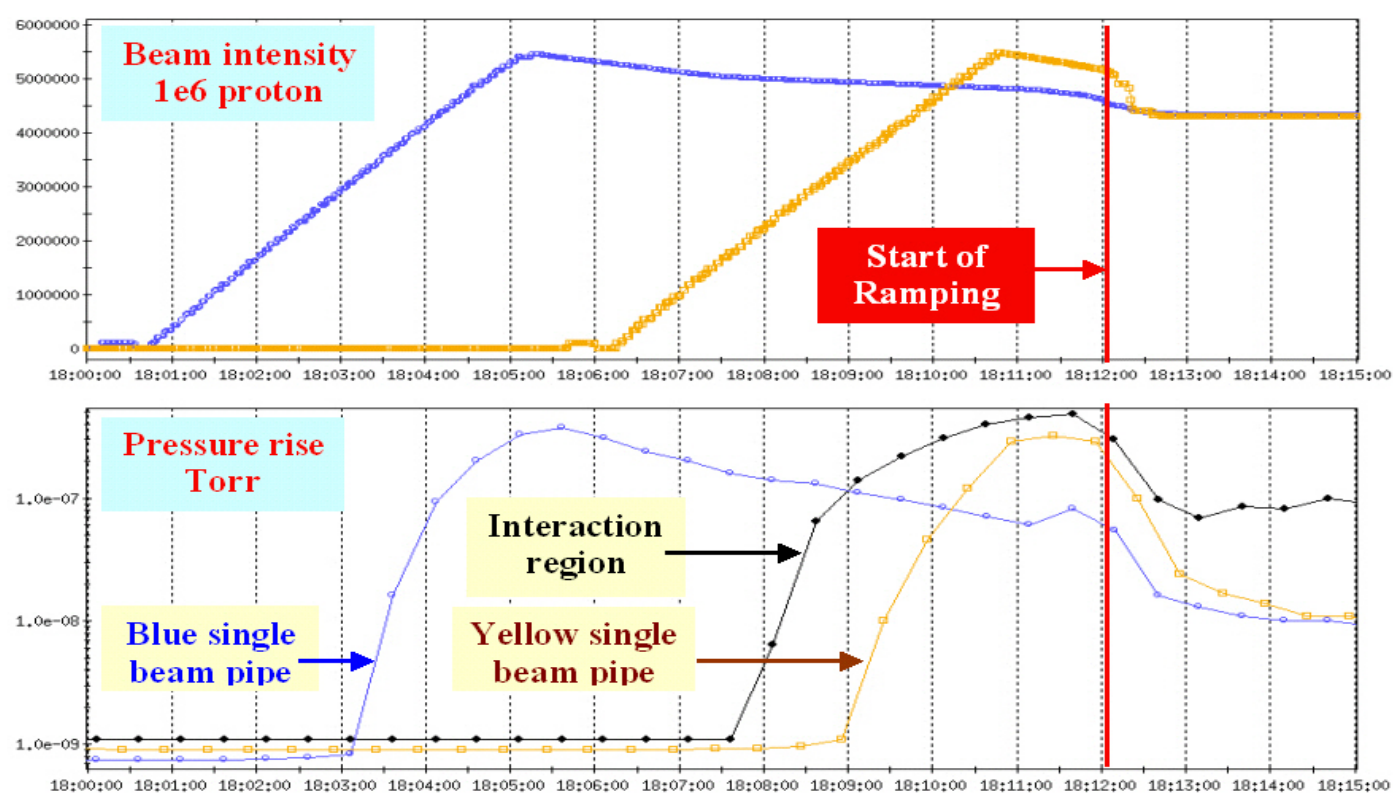

Fig.3: In proton run, once the ramp is started, pressure rise decreases at both the single beam straight section and interaction section 
This is explained as that once the ramp is started, the beam halo scraping is reduced because of the smaller transverse beam size. This makes the electrons more difficult to survive the bunch gap, since the scraping produced positive ions are reduced. In this way, the reduced electron multipacting results in lower pressure rise.

In Fig.4, it is shown that for a deuteron beam acceleration, the pressure started to rise once the ramp is started, and the pressure reached peak at the transition.
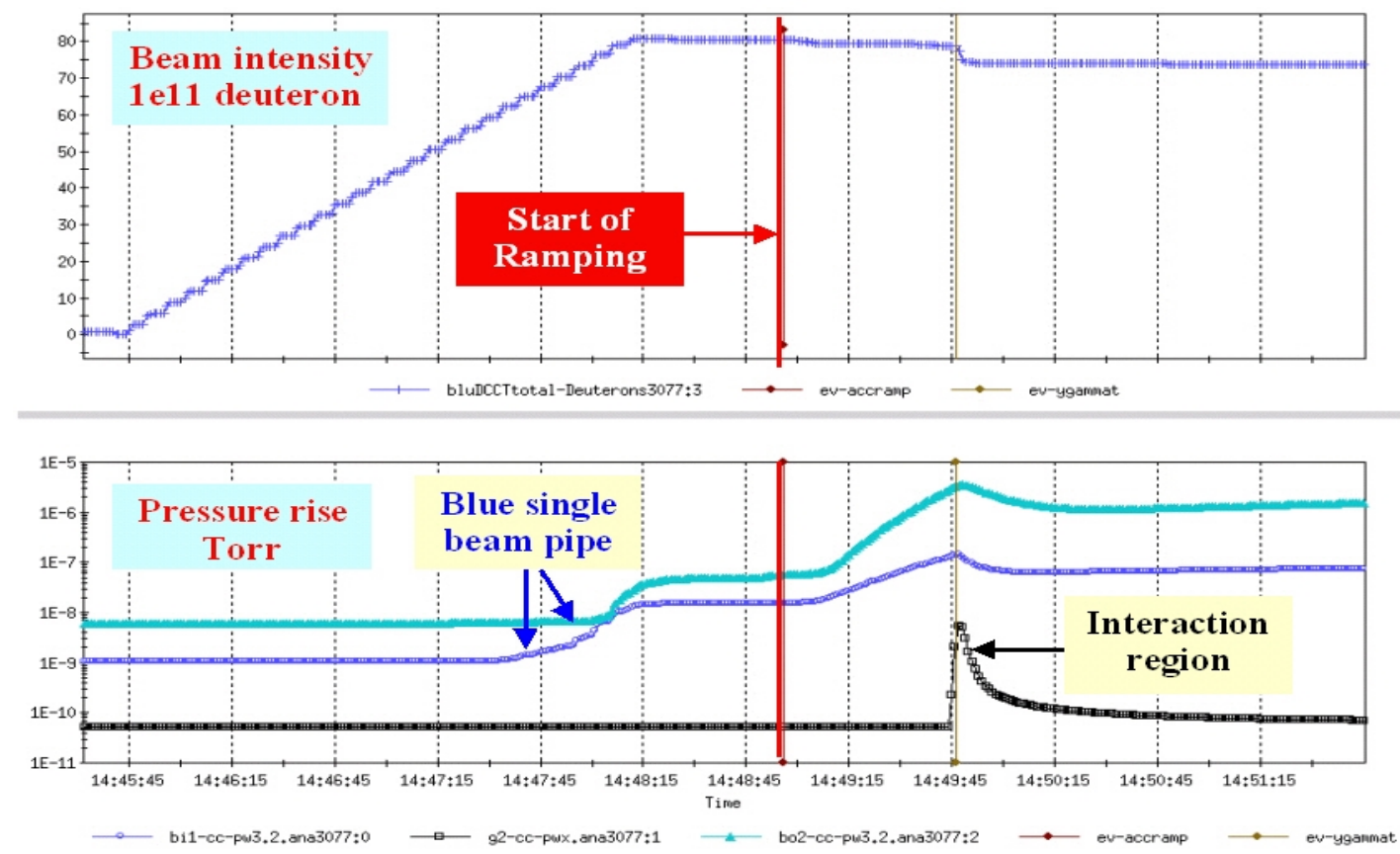

Fig 4: In dAu run, once the ramp is started, pressure rise increases at both the single beam straight section and interaction section Pressure rise reaches peak at the transition.

In both proton and deuteron accelerations, longitudinal and transverse beam sizes are decreasing once the ramp is started. The beam momentum spread is also reduced for proton acceleration, but it is increased for deuteron and gold run during the early part of the ramp, and peaked at the transition. Therefore, in deuteron and gold acceleration, the larger beam momentum spread may increase the beam halo scraping, and hence causes pressure rise, due to the ion desorption.

As a brief conclusion, to improve RHIC pressure rise situation, not only the SEY, but also the electron and ion desorption rates need to be reduced.

\section{NEG coating}

Typically, NEG with the composition $\mathrm{Ti}_{30} \mathrm{Zr}_{30} \mathrm{~V}_{40}$ is coated by sputtering with 1 to $2 \mu \mathrm{m}$ thickness on the chamber wall. Baking in vacuum at a few hundred degree $\mathrm{C}$ is needed to activate the getter. The getter film surface will saturate after certain amount of adsorption, and reactivation is required. This kind of re-activation and the air-exposure/activation, which is called a venting cycle, cause aging of the NEG coating.

Disregard many details, the following represents general function and property of NEG coating. 
1. With the help of ion pumps, the ultimate pressure of low $10^{-12}$ Torr can be reached in a typical vacuum chamber.

2. After activation, initial pumping speed for $\mathrm{H}_{2}, \mathrm{CO}$, and $\mathrm{N}_{2}$ is in the order of $10^{3} \ell \mathrm{s}^{-1} \mathrm{~m}^{-1}$. Pumping speed may rapidly reduce to less than half, then becomes stabilized.

3. Pumping speed decreases by two decades after the adsorption of 0.1 Torr $\ell \mathrm{m}^{-1}$ of $\mathrm{CO}$.

4. Activation of $\mathrm{Ti}_{30} \mathrm{Zr}_{30} \mathrm{~V}_{40}$ coating requires 1 hour at 250 degree $\mathrm{C}, 5$ hours at 200 degree $\mathrm{C}$, and 24 hours at 180 degree $\mathrm{C}$.

5. Due to aging effect, initial pumping speed decreases by one decade after 10 venting cycles.

6. A $\mathrm{Ti}_{30} \mathrm{Zr}_{30} \mathrm{~V}_{40}$ coating with $5 \mu \mathrm{m}$ thickness may undergo 50 venting cycles, with less than half pumping speed, even with the baking temperature increased from 180 degree $\mathrm{C}$ to 350 degree $\mathrm{C}$, at the end.

Several aspects of the NEG coating will be reviewed. These are secondary electron yield, electron stimulated desorption rate, ion desorption rate, pumping capability, activation, and aging.

\section{Secondary electron yield}

For un-activated NEG coating, the SEY is about 2, which is similar to the one of typical stainless steel surface. After 2 hours of 200 degree $\mathrm{C}$ baking in vacuum, the SEY is reduced to 1.1. The peak yield energy of the primary electrons changes very little, around 300 to $350 \mathrm{eV}$. SEY increases very little, to 1.2 , for the saturated surface.

A recent study at CERN SPS confirmed that NEG coating helps to suppress electron multipacting. In 6-meter NEG coated beam pipe, no electron signal was detected, while stainless steel chamber showed usual strong electron multipacting [5].

\section{Electron stimulated desorption rate}

In a measurement using $500 \mathrm{eV}$ electrons as projectile, the gas desorption rate of a NEG coated surface is compared with the stainless steel surface baked at 300 degree $\mathrm{C}$ in two hours [4]. Desorption rate of $\mathrm{H}_{2}$ is $10^{-2}$ for steel, and $2 \times 10^{-4}$ for activated NEG coating. Desorption rate of $\mathrm{CO}$ is $8 \times 10^{-3}$ for steel, and $10^{-4}$ for activated NEG coating. In general, the electron desorption rate is reduced up to a factor of 100 for NEG coating.

\section{Ion gas desorption rate}

In the study at CERN LINAC3 [6], a $\mathrm{Ti}_{30} \mathrm{Zr}_{20} \mathrm{~V}_{50}$ coated pipe, with thickness of $1.5 \mu \mathrm{m}$, was tested using $4.2 \mathrm{MeV} / \mathrm{n} \mathrm{Pb}^{53+}$ ions as projectile. The test chamber is 1.4 meters long, and the heavy ion beam was injected in very shallow angle of 89.2 degrees.

For NEG coated chamber activated at 300 degree $\mathrm{C}$, the ratio of desorption rate of NEG coating over the stainless steel surface is 0.12 for $\mathrm{H}_{2}, 0.04$ for $\mathrm{CO}$, and 0.1 for $\mathrm{CO}_{2}$. In total, the desorption rate of NEG coated surface is about 0.06 of the steel.

The measurement of the desorption rate is not straightforward, because of high pumping speed of the NEG coated chamber. A single shot beam is injected, and the peak pressure rise is carefully compared with the mean pressure rise before the impact of the lead ions.

\section{Pumping capability}

Since rare gases, e.g., Ar, He, and light hydrocarbon, e.g., $\mathrm{CH}_{4}$, do not react with NEG, they are not pumped. Fortunately, these gases are almost non-detectable in the RHIC pressure rise. Therefore, existing RHIC ion pumps can easily handle these gas components. 
For most concerned gases, such as $\mathrm{CO}, \mathrm{H}_{2}, \mathrm{CO}_{2}$, etc., NEG coating typically provides initial pumping speed of $10^{3} \mathrm{ls}^{-1} \mathrm{~m}^{-1}$. Hydrogen gas diffuses into the bulk, therefore, it does not affect the pumping of other gases. On the other hand, $\mathrm{CO}$ inhibits completely, and $\mathrm{N}_{2}$ intermediately, the pumping of other gases. Adsorption of $\mathrm{CO}$ of 0.1 Torr $\ell \mathrm{m}^{-1}$ reduces the initial pumping speed by a factor of 100 , to about $10 \ell \mathrm{s}^{-1} \mathrm{~m}^{-1}$. If this level of pumping is not acceptable, then for typical RHIC equilibrium pressure, activation is needed every 100 days.

In the measurement at the LINAC3 [6], for single beam shot injection, time constant of pressure reduction is in the order of a few tenths of second, as compared with stainless steel pipe with normal pumping of many seconds.

\section{Activation}

The activation is an important issue for NEG coating at RHIC interaction section. For example, the experiments STAR and BRAHMS have the beryllium beam pipes with aluminum extensions, which cannot be baked to high temperature. Special attention is needed if there is interest to apply NEG coating there.

The lowest activation temperature for $\mathrm{Ti}_{30} \mathrm{Zr}_{30} \mathrm{~V}_{40}$ film is at 180 degree $\mathrm{C}$, and higher temperature brings in better performance. In reality, baking temperature higher than 180 degree $\mathrm{C}$ may be needed. One example is shown in [6]. For two pieces of NEG coated pipe, the one treated by 300 degree $C$ activation is much better than the 200 degree $C$ which only improved ion desorption by a factor of 2 , compared with the steel.

The activation frequency is also of concern, partly because of the existing data is not very complete, and partly because of the cost, time, and labor concerns.

\section{Aging}

Once the coated surface is saturated, activation can diffuse the oxygen and carbon into the bulk of getter. Therefore, the getter volume will be progressively loaded with oxygen and carbon, which implies aging. The air exposure, followed by an activation, introduces even larger load, therefore, aging is usually accounted by how many venting cycles the coating can last. As mentioned earlier, a NEG film with thick coating may last up to 50 venting cycles.

Pumping and other properties of the NEG coating are affected by the aging. A typical example is that in 10 venting cycles, the pumping speed is reduced by a factor of 10 [4], under same activation temperature. With increased temperature, the pumping speed will be restored somewhat.

\section{NEG coating concerns at RHIC}

NEG coating has shown significant reduction in SEY. Activation at 200 degree $\mathrm{C}$ in 2 hours reduces SEY from 2.0 to 1.1. For comparison, TiN coating can reduce the SEY to about 1.6. In SPS 2002 beam scrubbing of 10 days, SEY was also reduced to about 1.6, and electron signal was almost disappeared.

It is remarkable that the SEY increases very little to 1.2, after the coating is saturated. Therefore, it is not surprised to find that in SPS test of 6 meter NEG coated pipe, no electron signal was observed [5].

There are two possible sources of electrons that cause pressure rise at RHIC. The first is the electron cloud, where electrons are produced during the electron multipacting. These electrons are then kicked to the wall by passing bunches, and cause pressure rise by electron desorption. The 
second one is that during the beam loss and/or beam halo scraping, abundant electrons are produced as by-product, especially for heavy ion beam. These electrons will also contribute to pressure rise. Therefore, electron desorption rate needs to be reduced, even the SEY is reduced and electron multipacting is under control.

For electron desorption rate, NEG coating provides up to a factor of 100 reduction. In [4], it is also shown that under $500 \mathrm{eV}$ electron bombardment, there is more than 3 orders of magnitude reduction in pressure rise for activated NEG coating, compared with baked steel chamber.

Compared with the confidence in the reduction of SEY and electron stimulated desorption rate, current knowledge of NEG coating ion desorption rate is less clear, and might be less promising. The reduction of ion desorption rate of NEG coating seems obvious, but only by a factor of 10 . Since NEG coating's large pumping speed, it is not straightforward to identify the ion desorption contribution, therefore, it is possible that even this ion desorption reduction is overestimated.

A measurement using saturated NEG coated chamber may generate better estimate of the ion desorption rate. Also, if the measurement can be extended to different ion species, energy, impact angle, and ion's charge state, it will help for more realistic evaluation of applying NEG coating at RHIC.

\section{V.Summary}

To solve the RHIC pressure rise problem, not only SEY, but also electron and ion desorption rates need to be reduced.

NEG coating has very good SEY reduction, beyond the TiN coating and the best beam scrubbing results so far. In addition, it provides up to a factor of 100 reduction in electron stimulated desorption rate. Therefore, NEG coating will help to suppress the electron multipacting and the electron desorption induced pressure rise.

On the other hand, experiment measurement shows that the improvement in ion desorption rate of NEG coating is only in the order of 10 . More measurements are needed.

High linear pumping of NEG coating will certainly benefit RHIC. Activation and aging problems need to be evaluated but are not the show stopper.

In next RHIC gold run, a test plan to install NEG coated chambers in several sections is presently under intense discussion.

\section{References}

1. C. Benvenuti, EPAC, p.200, 1998, and PAC, p.602, 2001.

2. S. Y. Zhang et al. PAC, 2003.

3. S.Y. Zhang and H.C. Hseuh, C-AD AP Tech Note 73, 2002.

4. P. Chiggiato, LHC-VAC/ChR/mpt, CERN, 2001.

5. J.M. Jimenez, PAC, 2003.

6. E. Mahner, PRAB, Volume 6, 013201, 2003. 